\title{
Appendix G
}

Theoretical analysis of whether a 2-fold increased risk of pH1N1 associated with prior seasonal influenza vaccination could be explained by trivalent inactivated influenza vaccine (TIV) effectively blocking the heterosubtypic cross-immunity provided by prior seasonal influenza infection 


\section{Appendix G. Can a two-fold increased risk of pandemic influenza A/H1N1 (pH1N1) associated with prior seasonal influenza vaccination be explained by vaccine effectively blocking the heterosubtypic cross-immunity provided by seasonal influenza infection?}

We have preliminarily explored this hypothesis on theoretical grounds. Based on the schematic below for pH1N1 in TIV vaccinated and unvaccinated groups taking into account prior seasonal influenza and assuming:

a) 100 people who received seasonal trivalent inactivated influenza vaccine (TIV VACCINATED)

b) 100 people who did not receive seasonal TIV [NOT TIV VACCINATED]

c) A seasonal influenza infection attack rate (AR) of $70 \%$ (single season or accumulated across seasons)

$>\mathrm{C}=70$;

$>\mathrm{A}=100-70=30$

d) A TIV vaccine effectiveness (VE) of $50 \%$ vs. (pH1N1 immunity-inducing) seasonal influenza infection

$>\mathrm{b}=\mathrm{VE} * \mathrm{C}=0.5 * 70=35$;

$>\mathrm{c}=(1-\mathrm{VE}) * \mathrm{C}=(1-0.5) * 70=35$;

$>\mathrm{a}=100-(\mathrm{b}+\mathrm{c})=100-(35+35)=30$

e) A $10 \% \mathrm{pH} 1 \mathrm{~N} 1$ attack rate (AR) during the spring/summer 2009 in Canada

$>\theta_{1}=0.10$

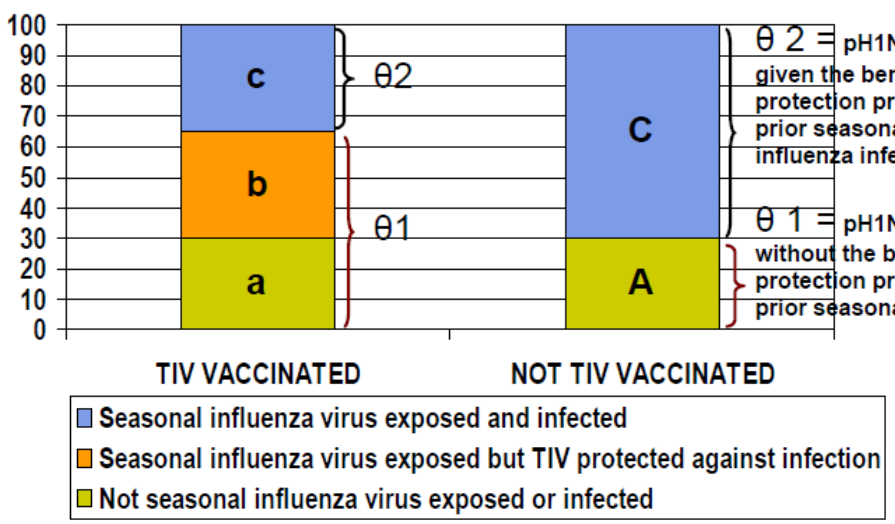

Then we can derive the required attack rate for $\mathrm{pH} 1 \mathrm{~N} 1$ infection $(\theta 2)$ to yield a risk ratio for $\mathrm{pH} 1 \mathrm{~N} 1$ infection of 2.0 for the vaccinated compared to the unvaccinated, based on the equation below for RR:

$$
R R_{p H 1 N_{1} \text { Vacc } / \text { Unvacc }}=\frac{\left[(\mathrm{a}+\mathrm{b})^{*} \theta 1+\left(c^{*} \theta 2\right)\right]}{\left(A^{*} \theta 1\right)+\left(C^{*} \theta 2\right)}
$$

Thus, $\quad 2=[(30+35) * 0.1+(35 * \theta 2)] /[30 * 0.1]+(70 * \theta 2)]$

$\theta 2=0.5 / 105=0.005$

This means that the $\mathrm{pH} 1 \mathrm{~N} 1$ attack rate would be reduced from $10 \%\left(\theta_{1}=0.10\right)$ in those without the benefit of prior seasonal infection to $0.5 \%\left(\theta_{2}=0.005\right)$ in those who had the postulated benefit of prior seasonal influenza infection - in other words, it would require that seasonal influenza infection provides cross-protection of $>95 \%$ against $\mathrm{pH} 1 \mathrm{~N} 1\left[\left(\theta_{1}-\theta_{2}\right) / \theta_{1}\right]$, which seems implausible given that the pandemic with assumed $10 \%$ attack rate occurred in that same immuno-epidemiologic context.

We can vary these assumptions, noting that plausibility is driven by prior seasonal influenza AR, seasonal influenza infection-induced cross-immunity to $\mathrm{pH} 1 \mathrm{~N} 1$, or TIV block of that and that the RR is unaffected by $\theta_{1}$.

If we assume a higher seasonal influenza attack rate $(95 \%)$ over several seasons and repeat annual vaccination with effectiveness in blocking that of $50 \%\left(C=95 ; A=5 ; a=5 ; b=47.5 ; c=47.5 ; \theta_{1}=0.10\right)$, then to achieve a relative risk of 2.0 would require $\theta 2=0.0298$ - in other words, a reduction in the pH1N1 AR from $10 \%$ in those without the benefit of seasonal infection to $3 \%$ in those who had benefited from prior seasonal infection. It may be debatable whether $70 \%$ cross-protection against $\mathrm{pH} 1 \mathrm{~N} 1\left[\left(\theta_{1^{-}} \theta_{2}\right) / \theta_{1}\right]$ could be afforded by seasonal influenza infection but this again seems unlikely.

With assumptions of seasonal influenza attack rates below 50\%, the hypothesis becomes completely unsupported (RR falls below 2) even assuming seasonal influenza infection induces $100 \%$ cross-protection against $\mathrm{pH} 1 \mathrm{~N} 1(\theta 2=0)$, unless TIV protection (VE) against that seasonal influenza infection is also assumed to be $100 \%$. 